P4.085 ASSESSING COMMUNICATION ON SEXUAL AND REPRODUCTIVE HEALTH ISSUES AMONG HIGH SCHOOL STUDENTS WITH THEIR PARENTS, BULLEN DISTRICT BENISHANGUL GUMUZ REGION, NORTH WEST ETHIOPIA

doi:10.1136/sextrans-2013-051184.0983

'D Gebrselassie, ${ }^{2} \mathrm{D}$ Gebselassie. ${ }^{1} \mathrm{MOH}$, Addis Ababa, Ethiopia, ${ }^{2} \mathrm{MOH}$, Addis Ababa univesrt public health dept, Addis Ababa, Ethiopia

Background "Adolescents "and "young people "are defined by WHO as the age group 10-19 years and 10-24 years, respectively. The health threats for adolescents today are predominantly behavioural rather than biomedical and more of today's adolescents are involved in health behaviours with potential for serious consequences. Hence, it is important to understand the exposure of school students to sexual and reproductive health knowledge and skills at different places including home.

Objective To assess the level and factors influencing communication between school students and parents on sexual and RH issues. Methods A cross-sectional survey using self-administered questionnaire and supplemented by focus group discussion was conducted in January 2006 at Bullen preparatory and high schools in northwest Ethiopia.

Results A total of 412 young people participated in the study. Of these, 284(68.9\%) were males and the rest 29.1\% females About $13 \%$ of the respondents were sexually active.

School was mentioned as the most common source of information for sexual and reproductive health issues followed by friends. Three hundred sixty two (88\%) respondents believed that it is important to discuss sexual and reproductive health issues/matters with parents. However, only 119 (28.9\%) of them discussed on two or more SRH topics with their parents. A high proportion of both male $(78 \%)$ and female (72\%) students preferred to discuss sexual and reproductive health issues with peers compared to less than $27 \%$ who prefer to discuss with parents.

Conclusions Although the majority of the students think discussion on sexual and reproductive health issues is important, they prefer to discuss with peers as this makes them more comfortable. Thus, it is essential to improve the sexual and Reproductive Health knowledge of school students to enhance peer influence positively and improve parent student communications through targeted family life education activities among students and parents.

\section{P4.086 CT TEST RESULTS AMONG PARTICIPANTS OF THE CHLAMYDIA SCREENING IMPLEMENTATION (CSI) AND ITS ASSOCIATION WITH SEXUAL BEHAVIOUR CHANGE}

doi:10.1136/sextrans-2013-051184.0984

L C Soetens, B H B van Benthem, E L M Op de Coul. National Institute of Public Health, Bilthoven, The Netherlands

Objective To assess the association between chlamydia (CT)-test results and subsequent sexual risk behaviour change in a register based Chlamydia Screening (CSI) programme consisting of four screening rounds.

Methods All participants in the CSI programme, who had completed questionnaires and corresponding CT-test results in $\geq 2$ rounds, were included. Measurements in round four were excluded, because of low participation rates. Sexual risk behaviour parameters contained the use of condoms, number of partners and having casual, concurrent or new partners. Generalised estimating equations (GEE) were used to assess the influence of the test result on the change in sexual risk behaviour in the subsequent round. Analysis were adjusted for sexual preference, having a steady or casual partner, age and number of rounds participated.

Results Of 9360 included participants, $80.5 \%(\mathrm{n}=7536)$ and $19.5 \%$ $(\mathrm{n}=1824)$ completed CT-testing and questionnaires in respectively two and three rounds. Across all rounds, a significant difference in change of sexual risk behaviour could be found: participants who tested CT-positive in the previous round more often reported reduced risk behaviour (more use of condoms with a casual partner (32.1\% vs $24.1 \%, p=0.018)$, less having sex with a casual partner $(21.7 \%$ vs $15.3 \%, p=0.001)$, lower number of sex partners $(38.5 \%$ vs $24.7 \%, \mathrm{p}<0.001)$, less having sex with concurrent partners $(15.3 \%$ vs $8.9 \%, p=0.005)$ and less having new sex partners $(21.0 \%$ vs $16.3 \%, p=0.010)$ ) compared to participants who tested CT-negative in the previous round. No significant difference in change of the use of condoms with a steady partner $(p=0.926)$ was found between participants who tested positive and participants who tested negative in the previous round.

Conclusion Sexual risk behaviour change was associated with previous CT-test results. Participants who tested CT-positive more often positively changed their sexual risk behaviour compared to participants with a negative test result in the previous round.

\section{P4.087 ARE BELGIAN STUDENTS BEING TESTED FOR SEXUALLY TRANSMITTED INFECTIONS, AND WHY (NOT)?}

doi:10.1136/sextrans-2013-051184.0985

'G Kayaert, 2 S A T Van den Eynde. 'Katholieke Hogeschool Leuven, Leuven, Belgium; ${ }^{2}$ Sensoa, Antwerp, Belgium

This research investigated to which extent students are tested for sexually transmittable infections (STIs). We also explored their motivations for getting tested (or not), as well as their knowledge and attitudes on STI-testing.

An online questionnaire was completed by 2274 students (between 19 and 23 years old), following a bachelor education in Leuven (Belgium)

The majority of the students (77\%) had sexual intercourse without a condom, during six months prior to the study. Within this group, a minority of $31 \%$ were tested on STIs at least once in their lifetime. A significant positive relationship existed between worrying about having contracted an STI and taking an STI test. Even within the subgroup of worried students $(16 \%)$, only a minority did get tested $(36 \%)$

A majority of students considered it "likely" to contract an STI when having unprotected sex with a new sex partner. An STI was expected to be painful, shameful, and even "terrible". Most students desired more information on STI testing.

Significant differences existed between men and women in key disadvantages of getting tested on STIs. Young women were reported embarrasment during the test and aversion for inspection of their genitals and/or questions about their sex life. Female respondents attached more importance to the gender of the doctor being female.

Young men were more bothered by practical issues, like the time and money an STI test would require. Male respondents attached less importance to an STI test as long as long as symptoms were absent.

We conclude that a substantial proportion of the students that would benefit from an STI test, do not get tested. More support to overcome practical and emotional barriers against STI-testing is required. There was a gender difference observed in the kind of support needed.

\section{P4.088 UNPROTECTED CASUAL SEXUAL CONTACT COMMON WITH BOTH LOCAL AND WESTERN PARTNERS AMONG DUTCH LONG-TERM TRAVELLERS TO (SUB)TROPICAL COUNTRIES}

doi:10.1136/sextrans-2013-051184.0986

'J Whelan, 'S Belderok, ${ }^{1,2} \mathrm{G}$ Sonder, ${ }^{1,2} \mathbf{A}$ van den Hoek. ${ }^{1}$ Public Health Service, Amsterdam, The Netherlands; ${ }^{2}$ Academic Medical Center, Department of Internal Medicine, Division of Infectious Diseases, Tropical Medicine and AIDS, Amsterdam, The Netherlands 